Interaction of a pulsating vortex rope with the local velocity field in a Francis turbine draft tube

This content has been downloaded from IOPscience. Please scroll down to see the full text.

2012 IOP Conf. Ser.: Earth Environ. Sci. 15032040

(http://iopscience.iop.org/1755-1315/15/3/032040)

View the table of contents for this issue, or go to the journal homepage for more

Download details:

IP Address: 128.178.4.9

This content was downloaded on 15/12/2014 at 14:53

Please note that terms and conditions apply. 


\title{
Interaction of a pulsating vortex rope with the local velocity field in a Francis turbine draft tube
}

\author{
A Müller, A Bullani, M Dreyer, S Roth, A Favrel, C Landry and F Avellan \\ EPFL Laboratory for hydraulic machines Avenue de Cour 33bis, 1007 Lausanne, \\ Switzerland \\ E-mail: andres.mueller@epfl.ch
}

\begin{abstract}
Acoustic resonances in Francis turbines often define undesirable limitations to their operating ranges at high load. The knowledge of the mechanisms governing the onset and the sustenance of these instabilities in the swirling flow leaving the runner is essential for the development of a reliable hydroacoustic model for the prediction of system stability. The present work seeks to study experimentally the unstable draft tube flow by conducting a series of measurements on a reduced Francis Turbine model. The key physical parameters and their interaction with the hydraulic and mechanical system are studied and quantified. In particular, the evolution of the axial and tangential velocity components in the draft tube cone is analysed by means of Laser Doppler Anemometry. Combined with the calculation of the instantaneous vortex rope volume based on flow visualization and the measurement of the pressure fluctuations, the nature of the auto-oscillation in the draft tube flow is investigated.
\end{abstract}

\section{Introduction}

Flow instabilities involving cavitation interact dynamically with the surrounding system and may excite acoustic and structural resonances. A problematic example of this surge phenomenon in turbomachines is observed in liquid propellant rockets in the 1960s [1]. This case is at the origin of the development of analytical models identifying the key parameters governing stability by Brennen et al. [2]. Further developments investigating the respective roles of the various model parameters and stability mappings are presented by Tsujimoto et al.[4]. The mass flow gain factor representing the excitation mass source is identified as a key player for stability. Auto-oscillation in diffusers of hydraulic machines and its cause is studied in particular by Chen et al.[7], Alligné et al.[8] and Dörfler et al.[10], choosing different approaches modelling the physics of the draft tube flow. Yonezawa et al. [11] identify the destabilizing effect of the conically shaped diffuser as the main cause for instability.

In a former paper[12] the mass flow gain factor was determined together with the cavitation compliance experimentally in a quasi-static approach by comparing different operating points. To gain better insight into the physics of the full load instability a local approach is chosen in order to study the evolution of various flow and system parameters for an unstable operating point at two different stream wise locations of the conically shaped draft tube cone. The measured quantities include the volume of the vortex rope, the wall pressure in the draft tube cone and the local axial and tangential velocity components. 


\section{Experimental test case}

The tests were performed on a reduced Francis turbine model of specific speed $=0.27$ on the EPFL test rig PF3. The top of Figure 1 shows the draft tube cone of the model with the two-component optical probe for the Laser Doppler Anemometry (LDA). The intersection of the laser beams is situated approximately $0.33 \cdot \mathrm{D}$ from the symmetry axis of the cone and marks the position of the control volume where the axial and tangential velocity components are measured. Two stream wise measurement positions are chosen and are henceforth referred to as position 1 and position 2 on an upper and lower level of the draft tube cone, respectively. The two pressure sensors for the wall pressure measurements are located on the same horizontal planes as the LDA control volumes, 0.39.D and 1.02.D downstream the runner outlet, respectively. Both pressure sensors share the same angular position in the draft tube cone. The bottom of Figure 1 shows a cut through the upper measurement plane as well as the position of the high speed camera for the flow visualization and the LED backlight.

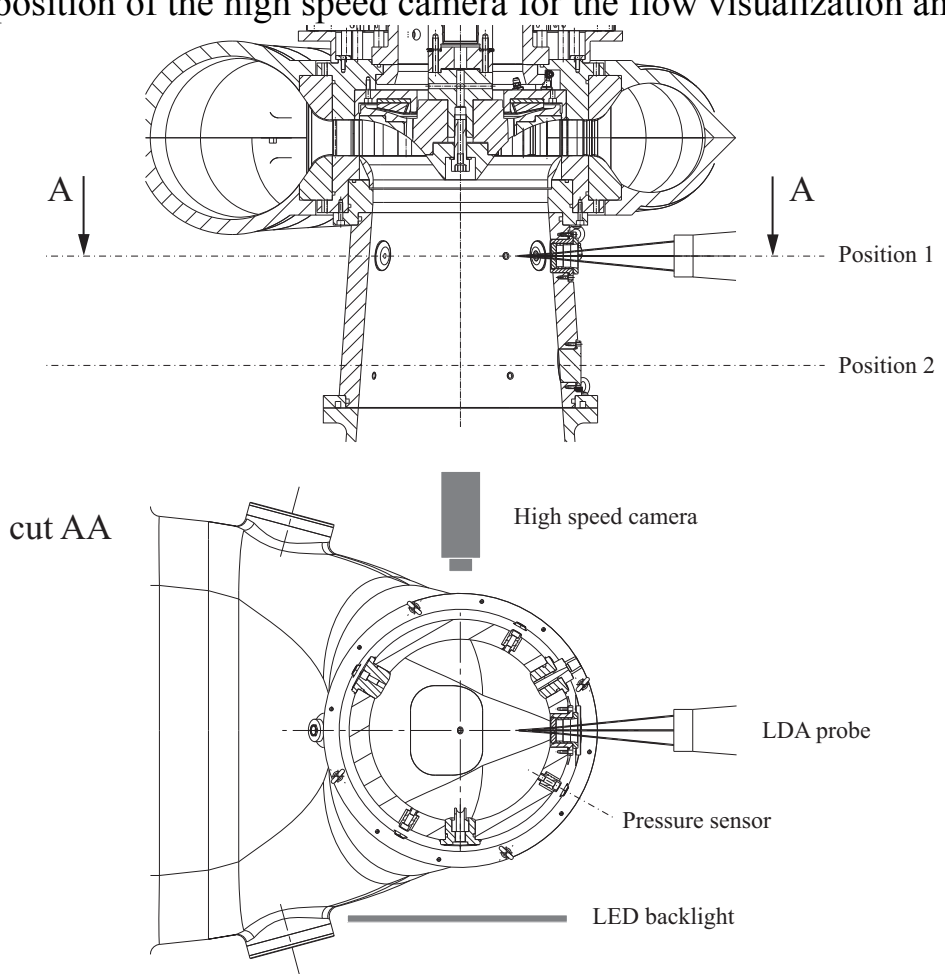

Figure 1. Instrumented draft tube cone with upper measurement plane

\section{Measurement parameters}

The measurements of the wall pressure, the velocity components and the visualization of the vortex rope are carried out simultaneously by sharing the same trigger between the different measurement systems. Piezo-restive pressure sensors are used to measure the wall pressure in the draft tube cone. The axial and circumferential velocity components are measured with a state of the art LDA system in non-coincidence mode and the water is seeded with hollow glass sphere particles. A high speed camera is used to film the vortex rope. The uniform LED backlight source provides good contrast between the liquid and gaseous phase. The operating conditions for the investigated instability are summarized in

\section{Table 1.}


Table 1. Summary of operating conditions

\begin{tabular}{ccccccc}
\hline \multirow{2}{*}{ Position } & $\begin{array}{c}\text { EM } \\
(\mathrm{J} \text { kg-1 })\end{array}$ & $\begin{array}{c}\text { QM } \\
(\mathrm{m} 3 \mathrm{~s}- \\
1)\end{array}$ & $\begin{array}{c}\text { NED } \\
(-)\end{array}$ & $\begin{array}{c}\text { QED } \\
(-)\end{array}$ & $(-)$ & $\begin{array}{c}\text { Fr } \\
(-)\end{array}$ \\
\hline 1 & 115.2 & 0.37 & 0.275 & 0.279 & 0.135 & 5.8 \\
2 & 113.1 & 0.36 & 0.277 & 0.275 & 0.118 & 5.8 \\
\hline
\end{tabular}

As it can be seen, the operating conditions for the two separately performed measurements at position 1 and 2, respectively, are subject to small variations. This is due to the fact that the stability conditions may slightly vary between two measurements and so certain operating conditions have to be tuned in order to provoke the instability.

\section{Results and analysis}

The pulsation of the vortex rope during one cycle of the instability is documented in Figure 2. The six different stages taken from a video recorded during the LDA measurement at position 1 are later identified with the letters A to F. The arrow indicates the location of the LDA measurement. For the calculation of the cavity volume based on its local diameter, the hypothesis of an axis-symmetric vortex rope is made. Unlike in [12], the use of a filter for the edge detection of the vortex rope was not necessary for the major part of the vortex rope due to the good contrast between the liquid and the gaseous phase and simple thresholding was applied. Optical deformations due to the conical shape of the draft tube are not taken into account. The accuracy of the volume's approximation varies furthermore with the rope shape. During the repetitive formation of the cavity, for instance, it is not possible to define clear edges, as can be seen in picture $\mathrm{C}$ of Figure 2.
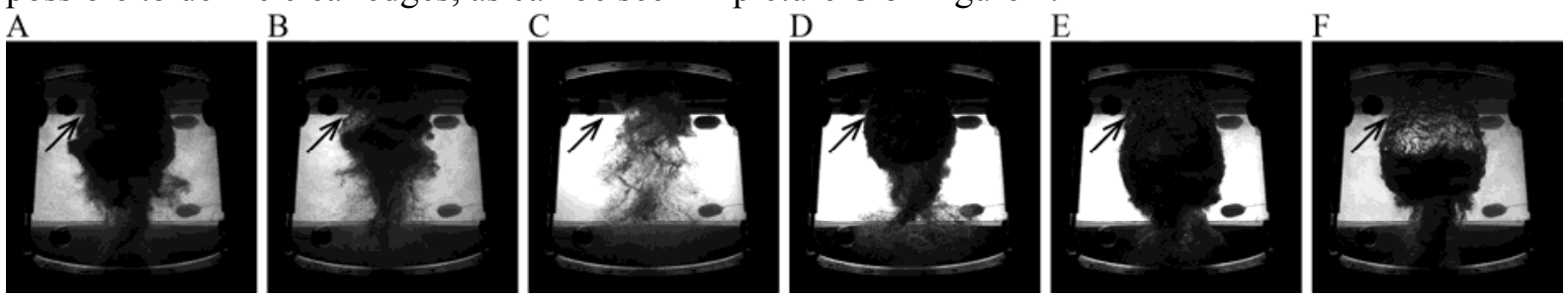

Figure 2. Vortex rope during one cycle of the full load instability

The evolution of the instantaneous vortex rope volume $V_{C}$, the wall pressure and the axial velocity $C_{m}$ in the meridional plane as well as the tangential velocity $C_{U}$ during four full cycles is shown in Figure 3 for position 1 . The void fraction is obtained by dividing the approximate instantaneous vortex rope volume $V_{C}$ by the total volume of the draft tube cone. The pressure coefficient $C_{P}$ is calculated by dividing the wall pressure signal by the water density and the overall specific energy $E^{M}$ at the given operating point. The axial velocity $C_{m}$ is made non-dimensional using the discharge velocity $C_{Q}$ of the flow across the section of the cone defined by the horizontal measurement plane. The tangential velocity $C_{U}$ is made non-dimensional using the circumferential speed $U$ of the runner at its outer downstream diameter.

As expected, the wall pressure rises to its maximum when the vortex rope volume decreases to its minimum. The meridional velocity component $C_{m}$ decreases when the vortex rope volume $V_{C}$ decreases and appears to behave symmetrically with respect to the pressure coefficient signal $C_{P}$. The tangential velocity component $C_{U}$ increases when the vortex rope volume drops and the pressure coefficient rises, however with a significant phase shift with respect to the meridional component $C_{m}$. 

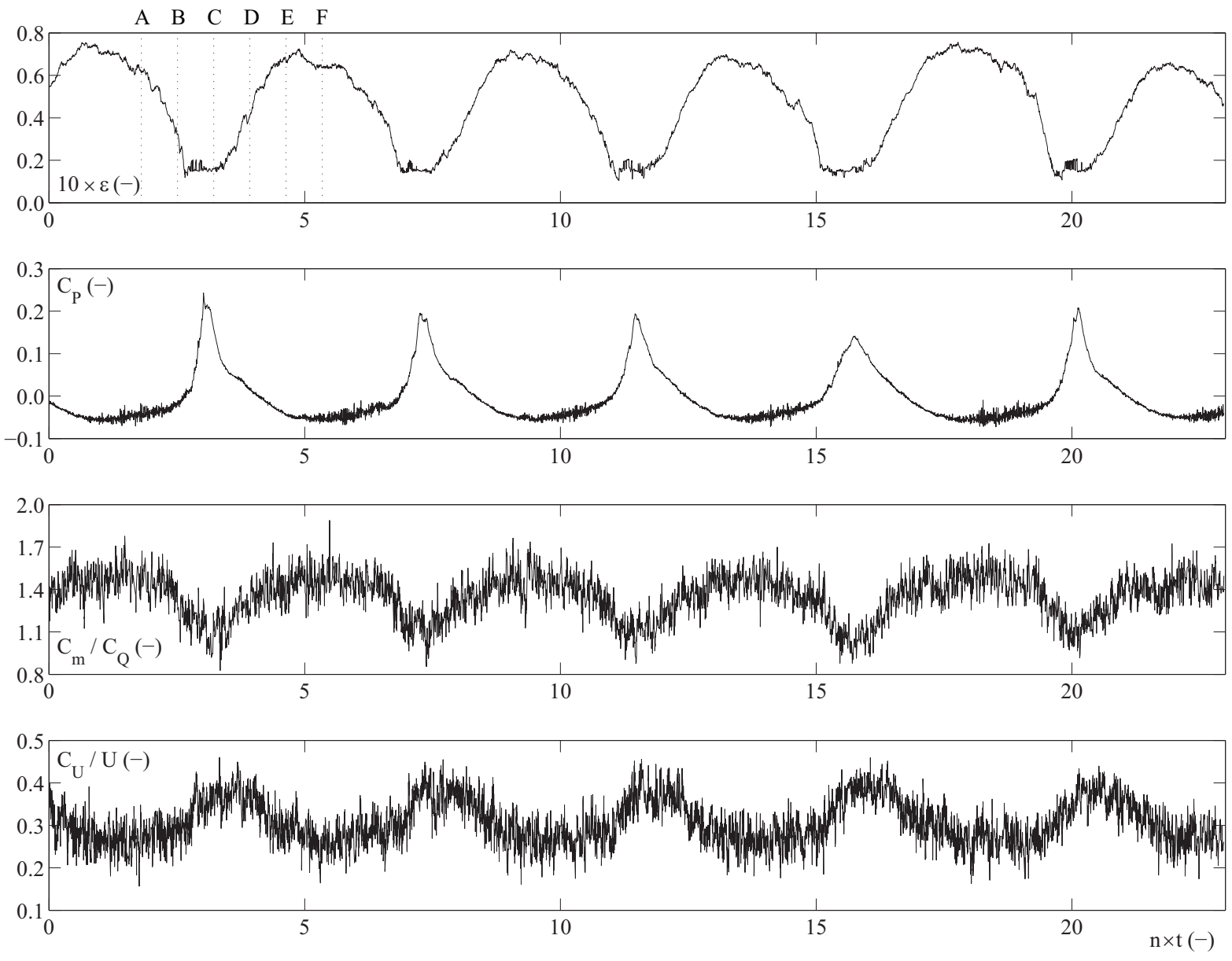

Figure 3. Approximate void fraction and raw signals of the pressure coefficient and the normalized meridional and axial velocity components in the draft tube cone as a function of the number of runner revolutions at position 1

In Figure 4 the $C_{m}$ and $C_{U}$ values are averaged with respect to the period of the wall pressure signal for both measurement positions 1, to the left, and 2, to the right. An analytic signal of $C_{P}$ is therefore calculated, consisting of a real part containing the original data and an imaginary part containing its Hilbert transform [13]. The argument of this analytic signal may be interpreted as an instant phase of the original data and the pressure signal can be divided into its different periods. The velocity signals are then split up individually so that every given fraction of the LDA signal would fit a single period of the $C_{P}$ signal. The recorded cycles of $C_{m}$ and $C_{U}$ are then superposed in one graph together with the superposed $C_{P}$ cycles. The calculated mean values of $C_{m}$ and $C_{U}$ are represented as straight thin lines in Figure 4 and the mean $C_{P}$ values are represented as straight bold lines, together with the standard deviations. One cycle is divided into 180 sub-cycles of 2 degrees each.

The axial speed $C_{m}$ at position 1 behaves almost symmetrically with respect to the pressure coefficient $C_{P}$ and reaches its minimum shortly after the maximum of $C_{P}$, as can be seen in the subplot to the top left of Figure 4. Further downstream at position 2 this effect seems to be cancelled out and only a small decrease in $C_{m}$ is detectable well after the pressure maximum, which is shown in the subplot to the top right. The tangential velocity $C_{U}$ is significantly elevated at both measurement positions with a phase shift compared to the $C_{P}$ signal. This is shown in the lower subplots of Figure 4, to the left for position 1 and to the right for position 2 . 

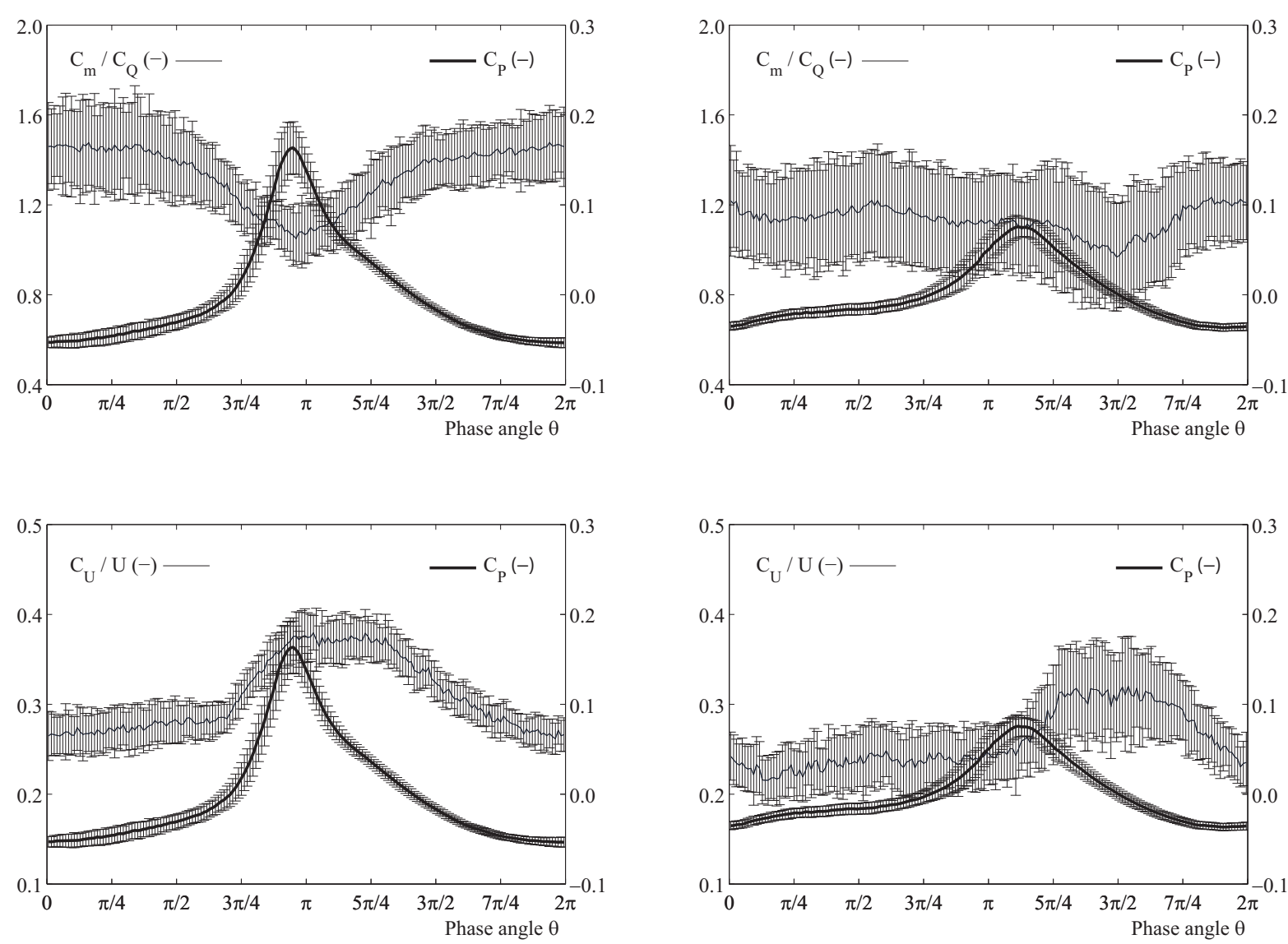

Figure 4. Phase averaged pressure coefficient as well as axial and tangential velocity components with mean and standard deviation values for position 1 to the left and position 2 to the right

\section{Conclusions and perspective}

The evolution of the axial and tangential velocity components is studied at an unstable full load operating point at two stream wise locations between the inner draft tube cone wall and the pulsating vortex rope. A periodical variation of the axial velocity component $C_{m}$ is observed at position 1 in form of a significant decrease, whereas this effect is flattened out downstream at position 2. The tangential velocity component $C_{U}$ periodically increases and decreases at both measurement positions with a delay compared to the $C_{P}$ signal.

The present analysis is limited to one radial position, $0.33 \cdot \mathrm{D}$ from the symmetry axis of the draft tube cone. However, LDA measurements were conducted at different radial positions. The resulting axial velocity components are to be used to calculate the instantaneous discharge at both vertical positions of the draft tube cone and hence the mass flow gain factor of the 1-D model proposed in [8]Furthermore, a total of twenty-one pressure sensors was distributed throughout the system at the upstream feeding pipe, the spiral case, the draft tube cone, the elbow and the diffuser. The corresponding pressure signals and their dependence on parameters such as the discharge factor $Q_{E D}$, the head factor $n_{E D}$ and the cavitation number is to be investigated. The instantaneous runner speed and torque were recorded simultaneously with the pressure and LDA signals in order to establish a hydraulic and mechanical energy balance at the instability. 


\section{Nomenclature}

\begin{tabular}{|c|c|c|c|}
\hline$C_{C}$ & Cavitation compliance $\left(\mathrm{m}^{2}\right)$ & $H$ & Hydraulic head (m) \\
\hline$C_{m}$ & Meridional (axial) velocity $\left(\mathrm{m} \cdot \mathrm{s}^{-1}\right)$ & $n$ & Runner rotational frequency $\left(\mathrm{s}^{-1}\right)$ \\
\hline$C_{P}$ & Pressure coefficient (-) & & Specific speed (-) \\
\hline$C_{U}$ & Circumferential (tangential) velocity $\left(\mathrm{m} \cdot \mathrm{s}^{-1}\right)$ & $N_{E D}$ & Speed factor $(-)$ \\
\hline$C_{Q}$ & Discharge velocity $\left(\mathrm{m} . \mathrm{s}^{-1}\right)$ & $Q^{M}$ & Model volumetric discharge $\left(\mathrm{m}^{3} \cdot \mathrm{s}^{-1}\right)$ \\
\hline$D$ & Runner outlet diameter (m) & $Q_{E D}$ & Discharge factor $(-)$ \\
\hline$E^{M}$ & Model specific energy ( ${\left.\mathrm{J} . \mathrm{kg}^{-1}\right)}^{-1}$ & $t$ & Time $(s)$ \\
\hline & Void fraction (-) & $U$ & Circumferential runner speed $\left(\mathrm{m} . \mathrm{s}^{-1}\right)$ \\
\hline $\mathrm{Fr}$ & Froude number (-) & $V_{C}$ & Cavity volume $\left(\mathrm{m}^{3}\right)$ \\
\hline$g$ & Gravitational acceleration $\left(\mathrm{m} \cdot \mathrm{s}^{-2}\right)$ & & Mass flow gain factor $(\mathrm{s})$ \\
\hline
\end{tabular}

\section{Acknowledgments}

This work is part of the HYSTAB research project, supported by EOS Holding and Andritz Hydro Ltd

\section{References}

[1] Rubin S 1966 J. of Spacecraft and rockets 3(8) 1188-1195

[2] Brennen C and Acosta A 1976 J. of Fluids Eng. 98 182-191

[3] Brennen C 1978 J. of Fluid Mech. 89 223-240

[4] Tsujimoto Y, Kamijo K and Yoshida Y 1993 J. of Fluids Eng. 115 135-141

[5] Tsujimoto Y, Watanabe S, Kamjio K and Yoshida Y 1996 J. of Fluids Eng. 118 (3) 589-594

[6] Tsujimoto Y, Yoshida Y, Watanabe S and Hashimoto T 1997 J. of Fluids Eng. 119 (4) 775-781

[7] Chen C, Nicolet C, Yonezawa K, Farhat M, Avellan F and Tsujimoto Y 2008 J. of Fluid Eng. 130041106

[8] Alligné S 2011 Forced and Self-oscillations of Hydraulic Systems induced by Cavitation Vortex Rope of Francis Turbines PhD Thesis (Switzerland:EPFL)

[9] Alligné S, Maruzewski P, Dinh T, Wang B, Fedorov A, Iosfin J and Avellan F 2010 Prediction of a Francis turbine prototype full load instability from investigations on a reduced scale model Proc. of the 25th IAHR Symp. on hydr. Machinery and Systems (Timisoara, Romania)

[10] Dörfler P, Keller M and Braun O, 2010, Francis full-load surge mechanism identified by unsteady 2-phase CFD, Proc. of the 25th IAHR Symp. on hydr. Machinery and Systems (Timisoara, Romania)

[11] Yonezawa K, Konishi D, Miyagawa K, Avellan F, Dörfler P and Tsujimoto Y 2012 Cavitation Surge in a Small Model Test Facility Simulating a Hydraulic Power Plant Int. J. of Fluid machinery and systems submitted for publication

[12] Müller A, Alligné S, Paraz F, Landry C and Avellan F 2011 Determination of Hydroacoustic Draft Tube Parameters by High Speed Visualization during Model Testing of a Francis Turbine Proc. of the 4th Int. Meet. on Cavitation and Dynamic Problems in Hydraulic Machinery and Systems( Belgrade, Serbia)

[13] Bendat J S and Piersol A G 2000 Random Data Analysis and Measurement Procedures, $3^{\text {rd }}$ ed (John Wiley \& Sons) 